\title{
FATIGUE FAILURE OF CORTICAL BONE SCREWS
}

\author{
Martin S. Zand, Steven A. Goldstein and Larry S. Matthews \\ Orthopaedic Biomechanics Laboratory, Department of Surgery, University of Michigan. \\ Ann Arbor, MI. U.S.A.
}

\begin{abstract}
An experimental study of the fatigue life of cortical bone screws under conditions which simulated in vivo usage was performed. The two most important factors influencing fatigue life were axial screw tension (the force normal of the plate to bone) and the cyclic shearing load. All screws failed at the root of the thread in the interface between the plate and the bone. A modified screw design effectively resisted fatigue under the described experimental conditions.
\end{abstract}

\section{INTRODUCTION}

Although fatigue fractures of cortical bone screws are a major factor in the failure of compression plate fixation, no implant specific study investigating the fatigue characteristics of cortical screws has been reported in the literature. The lack of a study focused on the design and fatigue failure modes of cortical bone screws prompted this research.

Three physical parameters determine the ability of a cortical screw to resist failure when subjected to cyclic shear stress through the compression plate during ambulation. These factors are the axial tension of the screw normal to the bone surface, the tension present in the compression plate, and the design of the cortical screw (Brad, 1980; Hayes and Perren, 1972; Perren et al., 1970). Some other factors which must necessarily be considered in plate and screw interactions are the torque necessary to achieve an adequate level of axial screw force, the coefficient of friction between the plate and bone (Hayes and Perren, 1972), cortical thickness and density, and the environment of implantation (Rostoker et al., 1978; Schatzker, 1975; Solar et al., 1979; Syrett and Davis, 1979). All of these secondary factors contribute to the effectiveness of fracture treatment with this method of internal fixation.

Axial screw tension has been shown to be the primary determinant of the physical stability and resistance to fatigue failure of a cortical bone screw (Koranyi et al., 1972). Because a surgeon cannot directly gage the axial force generated by a screw during insertion, he relies on the torque necessary to tighten the screw as an indicator of screw force. This implicit assumption of a relationship between torque and axial compression has been the subject of extensive investigation (Cordey et al., 1976; DeVito et al., 1981; Nunamaker and Perren, 1976). A single, reproducible, relationship between the compressive force generated by a screw, and the torque necessary to achieve that level of force, has not been documented for cortical bone in general. Instead, it is thought to vary with cortical thickness, screw type (self-tapping and non

Received for publication 1 November 1982. self-tapping), screw shaft diameter, and lubrication (Ansell and Scales, 1968: Schatzker et al., 1975; Uhtkoff, 1973).

Studies of the holding power of orthopaedic screws in cortical bone have consistently documented large values of axial tension when screws are used to hold compression plates to bone (Ansell and Scales, 1968; Cordey et al., 1975: DeVito et al, 1981; Hayes and Perren, 1972; Koranyi et al., 1972; Schatzker et al., 1975; Uhtkoff, 1973). These forces, normal to the bone surface, create a frictional resistance to plate movement. Hayes and Perren (1972) determined the coefficient of friction for stainless steel and cortical bone to be approximately 0.37 using sheep tibias. It is this coefficient of friction, along with axial screw tension, which maintains tension in the plate, and a corresponding compression across the fracture site. Increasing the normal force of the bone screw should, theoretically, increase the tensile load that the screws can maintain in the plate without it slipping (Brad, 1980). Preloaded tension in the plate, used for greater fracture and implant stability (Muller et al., 1979), combined with the forces of walking and muscular contraction. will cause the shaft of the screw to be subjected to cyclic shear loads. Varying the compression across the fracture site. and thus the tension in the plate, will vary the shear force acting on the screw; an increase in fracture compression will lead to a corresponding decrease in plate tension and screw shear. Such cyclic variation in the compressive forces across the fracture site occurs during normal walking.

One major advantage of compression plate fixation is that it permits early weight bearing. Although the dynamic forces which occur in the femoral and tibial shafts during ambulation have not been accurately quantified, it is possible to obtain a reasonable estimate of these forces from reported studies of force transmission across the knee and hip joints. Studies by Morrison (1970), Paul (1965) and Rydell (1973) have demonstrated forces of 1.2-4.0 times body weight present during normal walking. Bresler and Frankel (1971) externally instrumented the human leg during level walking, and found that the magnitude of the forces generated by such activity varied sinusoidally. Bechtol and Ferguson (1971) estimate that the im- 
plants will undergo 1-3 million cycles of loading over a one year period. Thus, the screws and plate will be subjected to repeated high magnitudes of cyclic loading during the period of fracture healing.

In the presence of cyclic loading, shearing forces, and corrosive body fluids (Solar et al., 1979; Syrett and Davis, 1979; Thompson et al., 1979; Wheeler and James, 1971), it is not surprising that the fatigue failure of standard cortical bone screws has been documented in the literature. Scales and Perry (1974), in comprehensive study of failed orthopaedic fixation devices, found that $6 \%$ of the cortical screws implanted during a ten year period fractured in normal use, in a mode characteristic of fatigue. Cahoon and Paxton (1970), as well as Proctor and Seaton (1974) examined unused cortical bone screws and found surface flaws caused by machining during the manufacture of the implants. They concluded that such flaws would significantly reduce the ability of the screws to resist all types of loading and that they were the most likely sites for fatigue fracture initiation. Only one study has addressed the problem of simulating implant fatigue. Wheeler and James (1971) conducted a study of the fatigue properties of 316 stainless steel in both air and Ringer's solution, concluding that the corrosive nature of Ringer's significantly reduced the fatigue life in the material.

The purpose of this study was to investigate the fatigue life and failure modes of cortical bone screws subjected to conditions which mimic in vivo usage.

\section{MATERIALS AND METHODS}

Thirty-seven human cadaver femora free from extreme osteopoenia or neoplasm were utilized. Their diaphyses were stripped of periosteum, cut into standardized $50 \mathrm{~mm}$ sections, and stored at $-20^{\circ} \mathrm{C}$. During testing, the bone sections were maintained at $22^{\circ} \mathrm{C}$ in a bath of lactated Ringer's solution.

To determine the relationship between torque and axial compression, the following procedure was used. A femur was prepared and mounted lengthwise in a stationary vise. A standard seven-hole Dynamic Compression Plate (DCP) was attached to the surface of the bone and a standard orthopaedic surgery drill guide was used to locate the screw hole sites. Two holes, $80 \mathrm{~mm}$ apart, were drilled with a $3.2 \mathrm{~mm}$ cortical drill bit, threaded using a $4.5 \mathrm{~mm}$ cortical tap, and cleaned of bone chips with Ringer's solution. The original plate was then removed, and a compression measuring apparatus (Fig. 1) was placed on the surface of the bone. The apparatus consisted of two specially machined sections from a DCP, two steel washers, and an $8 \mathrm{~mm}$ bolt force sensor transducer (Lebow, Model 3711). The force measuring system consisted of the piezoelectric transducer, a specially constructed charge amplifier, and a digitial volt meter for display. Twenty $4.5 \mathrm{~mm}$ cortical bone screws were used in this experiment. Each screw was inserted six different times into its hole. A calibrated strain gage

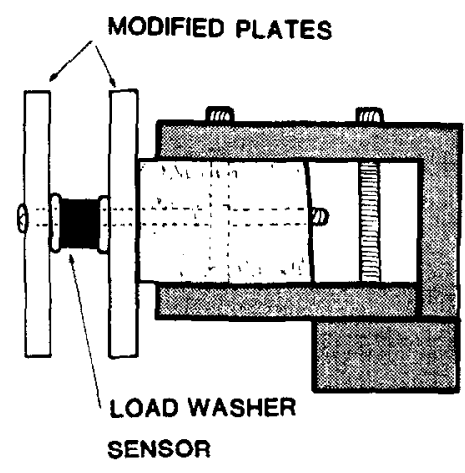

Fig. 1. $50 \mathrm{~mm}$ sections of bone were secured in a stationary vise, and compression of the plates to the bone was measured with a Lebow (Model 3711) bolt force sensor.

instrumented screwdriver was used to monitor insertional torque. Each screw was tightened until $440 \mathrm{~N}$ of axial compression was achieved, and the corresponding torque was recorded for each trial. The screw was kept in motion prior to attaining the desired level of compression, in order to avoid interference with the static frictional forces.

Using the data from the procedure described above, the following protocol was established for the fatigue testing program. The remaining bone specimens were cut into $50 \mathrm{~mm}$ sections. After preparation, a single specimen was mounted in a stationary vise (Fig. 2) on the bed of a fatigue testing machine (Sontag Model SF012-U). A standard seven-hole DCP was attached to the load arm of a separate vise and mounted on the vibrating platen of the machine. The compression plate and drill guide were used to drill a $3.2 \mathrm{~mm}$ hole with a cortical drill bit. The DCP was then removed, the hole threaded with a $4.5 \mathrm{~mm}$ cortical tap, and cleaned of bone chips with Ringer's solution. The compression measuring apparatus, as described above, was placed on the bone, and a $4.5 \mathrm{~mm}$ cortical screw was inserted into its hole three separate times. Each time, the torque necessary to achieve $400 \mathrm{~N}$ of axial plate compression was determined using the instrumented screwdriver. Before inserting the screw for testing, the compression measuring apparatus was removed, and the standard

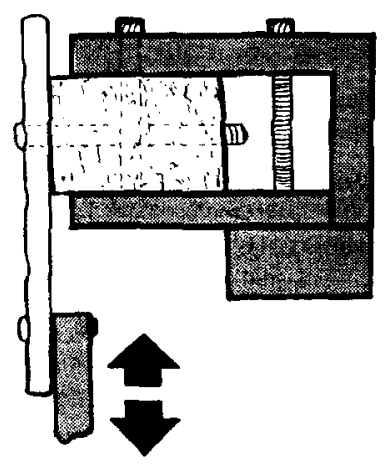

Fig. 2. The DCP plate was mounted to the loading platen of a Sontag (Model SF-012-u) fatigue testing machine. 
seven-hole DCP was reattached to the load arm of the fatigue machine. The screw was reinserted with the instrumented screwdriver using the third torque value previously measured as a control value, to achieve $440 \mathrm{~N}$ of axial compression. A cyclic shear load was applied to the screw through the plate at 1800 cycles per minute. It was assumed that the loading of the plate in tension produced a pure shear effect on the screw. The effect of bending due to a non-perfect plate-bone interface was felt to be negligible at high normal loads. Shear loads varied cyclically from $0 \mathrm{~N}$ to $711 \mathrm{~N}, 756 \mathrm{~N}$. $800 \mathrm{~N}, 822 \mathrm{~N}, 845 \mathrm{~N}, 867 \mathrm{~N}, 889 \mathrm{~N}$, or $993 \mathrm{~N}$, and was constantly measured by a piezoelectric force link mounted between the plate and the loading platen of the fatigue machine (Kristal, Model 9322). The signal from the force link was amplified (KIAG, Model 5001 Charge Amplifier) and displayed on an oscilloscope (Tektronix). Specimens were fatigued at $22^{\circ} \mathrm{C}$ while being constantly bathed in lactated Ringer's solution. Loading was conducted until 2.5 million cycles were endured, or until the screw failed. After testing, the bone and screw were removed from the apparatus. The screw was removed from the bone, and the cortical thickness at the insertion site was measured. Cortical screws were examined using both light and scanning electron microscopy.

A third and final study was conducted to determine the effect of varied levels of axial screw tension on fatigue life. The same procedure as described above was used to set up, insert and test the bone screws. All fatigue tests were conducted with a cyclic shear load which varied from $0 \mathrm{~N}$ to $933 \mathrm{~N}$. Screws were inserted with axial tensile loads of $488 \mathrm{~N}, 440 \mathrm{~N}, 418 \mathrm{~N}$ and $400 \mathrm{~N}$. Testing was conducted until either mechanical failure, functional failure*. or 2.5 million cycles were endured.

\section{RESULTS}

The data obtained from the insertional torque studies are illustrated in Fig. 3. The data as indicated was normalized by dividing the torque observed in any given trial by the first observed torque value. Insertional torque for all trials were in the range of 2.1 to $3.56 \mathrm{Nm}$. Final torque values varied widely for different bone sections, but after the third successive screw insertion, the value stabilized. This result made it possible to achieve stable and predictable insertional torque values on successive screw insertions (after the third) throughout the experiment.

A total of 42 standard $4.5 \mathrm{~mm}$ cortical bone screws were tested in fatigue loading. The relationship between shear stress and cycles endured to failure is illustrated in Fig. 4. Mechanical failure occurred in the range of $800-933 \mathrm{~N}$ of tensile load on the plate which corresponds to a nominal screw shear stress of ap-

* Functional failure was defined as screw-plate fixation failure.

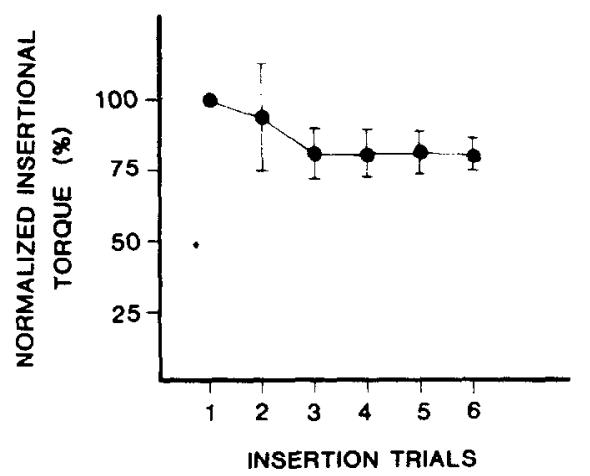

Fig. 3. The data from each series of insertional torque studies was normalized by expressing the successive insertional torques as a percent of the first value in the series. The insertional torque values were consistent for each screw after three successive insertions.

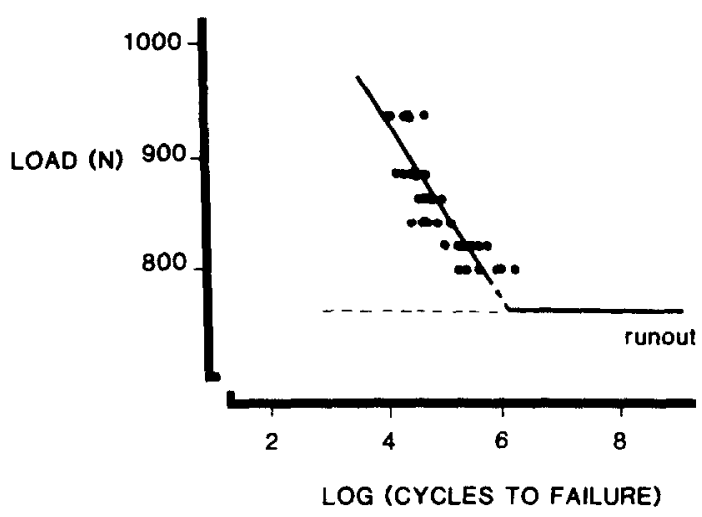

Fig. 4. The number of cycles to failure for standard cortical bone screws can be predicted from the tensile load on the plate when the normal compressive force of the bone is held constant.

proximately $261-305 \mathrm{MN} \mathrm{m}^{-2}$. Kunout, the condition where a screw endured over 2.5 million cycles of loading, occurred at a cyclic plate tension of $765 \mathrm{~N}$ or less.

Statistical analysis of the fatigue data at loads greater than $765 \mathrm{~N}$, using multiple least squared error regression and analysis of variance techniques illustrated several interesting findings.

The log (cycles to failure) could be predicted from a model involving the tensile load on the plate only. Cycles to failure were predicted from the following deterministic model:

$$
\log (\text { cyles to failurc })=14.03-0.011 \operatorname{load}(\mathrm{N}) \text {. }
$$

The relationship was significant at $p<0.001$ with a coefficient of regression $\left(r^{2}\right)$ of 0.70 , and the constants were significant at $p<0.001$. Predicted values are illustrated in Fig. 4. Non-linear models explained no more variance than this simple linear form.

Several other variables in the study illustrated trends toward satistical significance $(0.05<p<0.08)$. These variables include the bone sample to be used, and the interactions between bone segment relative position, 
and cortical thickness. The relative position of the bone segment was assigned on the following basis: each femur was divided into six $50 \mathrm{~mm}$ segments, the most proximal segment was assigned a value of 1 , and the most distal a value of 6 . While these variables explained very little additional variance in data, they re-emphasized the variability in bone properties among individual specimens.

All of the cortical screws which failed, did so at the second screw thread immediately beneath the head of the screw. Scanning electron microscopy revealed that such failures occurred at the root of the thread (Fig. 5), and that corrosion fatigue was present in $85^{\circ}{ }_{0}$ of the screws examined. The surface of the screw fractures in the shaft of the screw exhibited characteristics of both fatigue and brittle failure.

Examination of output from the force link mounted beneath the loading compression plate during testing revealed that the load-time history is an indicator of impending screw failure. The normal load-time history of a stable cortical screw during fatigue loading is a sinusoidal function. In $85^{\circ}$ of the screws which failed, however, that sine-wave pattern began to deform noticeably. Such characteristic deformation occurred after only $30^{\circ}$. of the fatigue life of the screw, and was an accurate predictor of mechanical failure. Some of the screws tested at lower load levels, although still intact after 2.5 million cycles, exhibited load-time histories characteristic of impending failure.

Based on the information obtained from the fatigue testing of normal $4.5 \mathrm{~mm}$ cortical screws, a new screw design was developed. The new design sought to improve fatigue resistance by strengthening the portion of the screw which failed in testing. The modified shaft $4.5 \mathrm{~mm}$ cortical bone screw (Fig. 6) differed from the standard screw only slightly. The first $9 \mathrm{~mm}$ of the screw shaft immediately beneath the head was left unthreaded, and retained the same outer diameter as the major diameter of the threads. All other material and design characteristics of the screw were left unchanged.

A total of 42 modified shaft cortical screws were tested using the same procedures and shearing force levels as the normal screws. A modification of the insertion procedure was necessary to accommodate the larger proximal shaft of the screw. This was accomplished by overdrilling the near cortex to $4.4 \mathrm{~mm}$ : all other procedures remained the same. Throughout the entire range of testing, not a single modified shaft cortical screw failed when subjected to fatigue loading. No evidence of impending failure in the load-time history was present in any of the screws tested.

A final experiment was done to investigate the influence of axial compression on the fatigue life of normal cortical bone screws. Five normal screws were tested at a constant level of cyclic shear stress for each level of axial load. Three distinct responses were observed. The screws inserted with $488 \mathrm{~N}$ of axial plate compression sustained 2.5 million cycles without failure; screws inserted with $440 \mathrm{~N}$ failed after 11.000 to 250,000 cycles; and screws inserted with $418 \mathrm{~N}$ and $400 \mathrm{~N}$ incurred functional failures after as few as 1000 cycles. Functional failure was the deformation or bending of the screw to such an extent that it was unable to hold the plate to the bone.

\section{DISCUSSION}

The torque-axial plate compression experiments provided a method of controlling screw force in the fatigue experiments without disturbing the bone/plate or plate/screw interfaces. Values obtained for these experiments for insertion torques are compatible with previous studies of clinical screw insertion torques and screw compression in which experienced orthopaedic surgeons tightened $4.5 \mathrm{~mm}$ cortical bone screws with torques of 2.65-9.71 Nm (Cordey et al., 1975, DeVito et al., 1981). Variation in the insertion torques is thought to be attributable to variance in cortical density and thickness, lubrication, and screw placement.

The data from the fatigue experiments revealed the classical fatigue relationship that increased loads lead to a shortened fatigue life. Although the statistical analysis of the data indicated that load is the primary determinant of the life of the screw, some variation in fatigue life for the same load levels can be attributed to differences in cortical thickness and bone segment location.

The loading values, screw insertion torques, and screw axial tension values were all within clinical tolerances as reported in the literature. All normal $4.5 \mathrm{~mm}$ cortical screws which failed in fatigue loading did so at the root of the second screw thread, proximal to the head of the screw. This area is the point immediately before the contact of the screw with the bone, the place where shear and bending forces would have the greatest effect, and the most likely spot for initiation of screw failure. Increasing the diameter of the screw decreases the shear stress for the same shear force as applied to a normal cortical screw. As demonstrated with the experiments on the modified shaft cortical screw, this significantly lengthens fatigue life. None of the modified shaft cortical screws so designed failed in testing.

The force normal-fatigue study indicated that axial plate compression and therefore friction play a key role in the stability of the cortical screw. Low values of axial compression, leading to low frictional forces between plate and bone, produced functional bending failures. High normal forces increased the resistance of the plate to movement, and thus increased screw fatigue life. In light of these data, and the basic principles of frictional interactions, it is appropriate to suggest that the same resistance to movement could be achieved by maintaining the axial compression of the screw constant, and increasing the frictional forces between plate and bone.

Previous studies of cortical screw design have 


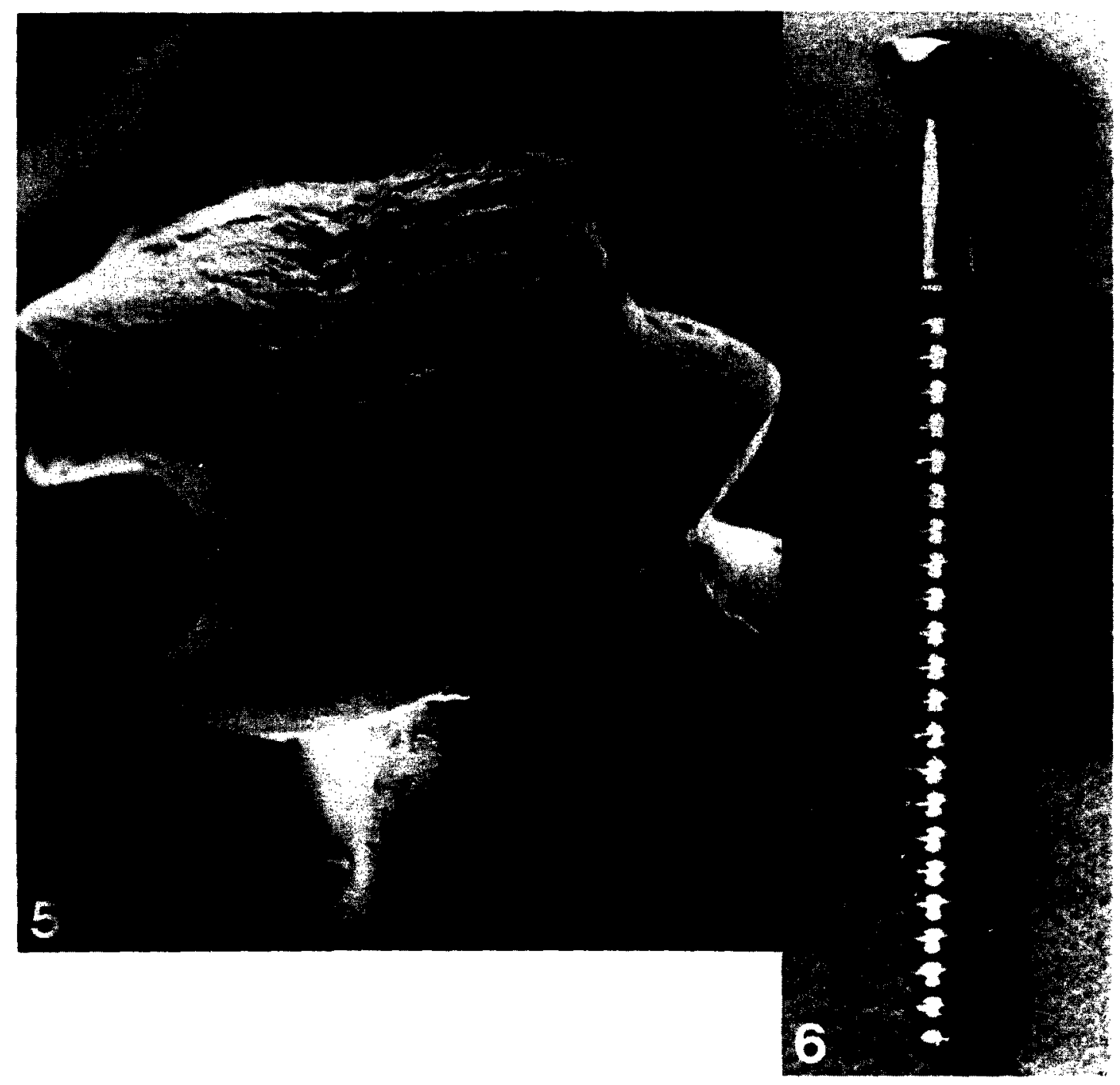

Fig. 5. Scanning electron microscopy of a failed screw.

Fig. 6. A modified cortical bone screw. 
suggested that holding power is one criteria for evaluating screw function (Schatzker et al. 1975; Ansell and Scales, 1968). Although pullout strength and holding power are important with respect to stripping of the cortical bone threads during screw insertion, a more important indicator of functional stability of the cortical bone screw may be fatigue testing. The ideal cortical screw, then, would be one which combined insertion stability in the form of holding power with fatigue resistance. This might be accomplished by threading the proximal end of the modified shaft screw with a half formed thread.

\section{CONCLUSIONS}

1. A direct relationship between applied torque and axial screw tension exists in screw insertion.

2. With a constant force normal holding a plate against bone, applied cyclic shearing load is the primary determinant of fatigue life.

3. Increasing the plate force normal increases the fatigue life of cortical bone screws.

4. Cortical screws used in compression plating which fail in fatigue, will do so at the root of the thread in the interface area between the plate and the bone.

\section{REFERENCES}

Ansell, R. H. and Scales, J. T. (1968) A study of some factors which affect the strength of screws and their insertion and holding power in bone. J. Biomech. 1, 279-302:

Brad, J. (1980) The concepts and terms of mechanics. Clin. Orthop. 146, 9-17.

Bresler, B. and Frankel, J. D. (1980) The forces and moments in the leg during walking. Trans. Am. Soc. mech. Engrs 1, $27-36$.

Brettle, J. (1970) A survey of the literature on metallic surgical implants. Injury 2, 26-39.

Brettle, J. and Huges, M. (1970) A metallurgical examination of surgical implants which have failed in service. Injury 2 , 143-154.

Burke. J. (Editor) (1977) Risk and Failure Analysis for Improved Performance Reliability. Plenum Press, New York.

Cahoon, J. R. and Paxton, H. W. (1968) A metallurgical analysis of failed orthopaedic implants. J. Biomed. mat. Res. 2, 1-22.

Cahoon, J. R. and Paxton, H. W. (1968) A metallurgical survey of current orthopaedic implants. J. Biomed. mat. Res. 2, 1-22.

Carter, D. R. and Hayes, W. C. (1976) Fatigue life of compact bone: 1. Effects of stress amplitude, temperature and density. J. Biomech. 9, 27-33.

Cauzaud, R. (1953) Fatigue of Metals. Philosophical Library Publishers, New York.

Clemens, D. et al. (1979) The influence of grain size on the fatigue behavior of annealed $316 \mathrm{LVM}$ stainless steel. J. Biomed. mat. Res. 13, 337-341.

Cordey, J. et al. (1976) The control of torque applied to bone screws used by experienced surgeons simulating internal fixation with plates. In Biomechanics $\boldsymbol{V}-\boldsymbol{B}$. International Series on Biomechanics p. 000, University Park Press, London.

Croquillet, B. et al. (1979) Influence of cold working on fatigue behavior of stainless steels used for prosthesis: application to the study of wires with small sections. J. Biomed. mat. Res. 13, 657-668.

DeVito, P., Rivlin, M., Price, D., Matthews, L. and Goldstein, S. (1981) Friction as a major factor in plate and screw fracture fixation stability: the design of a new plate surface. Ortho. Trans. (presented at the 1982 meeting of the Orthopaedic Research Society).

Evans, F. G. (1957) Stress and Strain in Bones. Thomas. Springield.

Ferguson, A. B. and Brown, T. D. (1980) Mechanical property distributions in the cancellous bone of the human femur. Acta orthop. scand. 51, 429-437.

Hayes, W. C. and Perren, S. M. (1972) Plate-bone friction in the compression fixation of fractures. Clin. Orthop. 89, 236-240.

Koranyi, B. S. et al. (1972) The holding power of orthopaedic screws in bone. Clin. Orthop. 72, 283-286.

Little, R. E. and Jebe, E. H. (1975) Statistical Design of Fatigue Experiments. John Wiley, New York.

Madayag, A. F. (1969) Metal Fatigue: Theory and Design. John Wiley, New York.

Malone, C. B. et al. (1955) Bone strength before and after removal of unthreaded and threaded bone screws. Clin. Orthop. 6, 259-260.

Mather, B. S. (1967) The symmetry of the mechanical properties of the human femur. J. Surg. Res. 7, 22-33.

Morrison, J. B. (1970) The mechanics of the knee joint in relation to normal walking. $J$. Biomech. 2, 51-61.

Muller, M. E. et al. (1979) Technique of Internal Fixation of Fractures. Springer-Verlag, Berlin.

Nunamaker, D. and Perren, S. M. (1976) Force measurements in screw fixation. J. Biomech. 9, 669-675.

Paul, J. P. (1965) Bioengineering studies of the forces transmitted by joints-11. Biomechanics and Related Bioengineering Topics (Ed. R. M. Kenedi), p. 369. Pergamon Press, Oxford.

Perren, S. M. et al. (1975) Biomechanics of fracture healing after internal fixation. Surg. Ann. 361-390.

Perren, S. M. et al. (1970) A dynamic compression plate in cortical bone healing. Acta orthop. scand. suppl. 126, $31-41$.

Proctor, R. P. (1968) An evaluation of the quality of stainless steel surgical implants. Injury 8, 102-109.

Proctor and Seaton (1974).

Rostoker et al. (1978) Evaluation of couple/crevice corrosion by prosthetic alloys under in vivo conditions. J. Biomed. mat. Res. 12, 823-829.

Rydell, N. (1973) Biomechanics of the hip joint. Clin. Orthop. 92, 6-25.

Scales. J. T. and Perry, P. (1974) Screw and plate devices in the fixation of fractures: a ten year survey. Acta orthop. belg. 40 , 836-845.

Schatzker, J. et al. (1975) The effect of movement on the holding power of screws in bone. Clin. Orthop. 111, 257-262.

Schatzker, J. (1975) The holding power of orthopaedic screws in vivo. Clin. Orthop. 108, 115-126.

Schatzker, J. et al. (1975) The reaction of cortical bone to compression by screw threads. Clin. Orthop. 111, 263-265.

Solar, R. J. et al. (1979) In vitro corrosion testing of titanium surgical implant alloys: an approach to understanding titanium release from implants. J. Biomed. mat. Res. 13, 217-250.

Syrett, B. C. and Davis, E. E. (1979) In vivo evaluation of a high-ductility stainless steel for use in surgical implants. J. Biomed. mat. Res. 13, 543-556.

Thompson et al (1979)

Uhtkoff, H. K. (1973) Mechanical factors influencing the holding power of screws in compact bone. J. Bone Jt Surg. 55B, 633-639.

Wheeler, K. R. and James, L. A. (1971) Fatigue behavior of type 316 stainless steel under simulated body conditions. J. Biomed. mat. Res. 5, 267-281. 